\title{
Health Related Quality of Life and Psychological Parameters in Different Polycystic Ovary Syndrome Phenotypes: A Comparative Cross-Sectional Study
}

\section{Fatemeh Bahadori}

Tarbiat Modares University Faculty of Medical Sciences

shahideh Jahanian Sadatmahalleh ( $\sim$ Shahideh.Jahanian@modares.ac.ir)

Tarbiat Modares University Faculty of Medical Sciences https://orcid.org/0000-0002-7006-8487

Negin Mirzaei

Tarbiat Modares University Faculty of Medical Sciences

\section{Research}

Keywords: polycystic ovary syndrome phenotypes, quality of life, anxiety, depression, health related quality of life

Posted Date: December 11th, 2020

DOI: https://doi.org/10.21203/rs.3.rs-125235/v1

License: (c) (1) This work is licensed under a Creative Commons Attribution 4.0 International License. Read Full License

Version of Record: A version of this preprint was published at Journal of Ovarian Research on April 24th, 2021. See the published version at https://doi.org/10.1186/s13048-021-00811-2. 


\section{Abstract}

Background: Polycystic ovary syndrome (PCOS) is associated with high levels of phsychological implications and detriments to quality of life (QoL). The aim of this study was to assess the quality of life (QoL), health related quality of life (HRQoL), depression, and anxiety in Iranian women with different PCOS phenotypes.

Methods: The observational, cross-sectional study was carried out on 239 PCOS women PCOS which had been classified on the basis of Rotterdam criteria into four categories. Then they were classified into four groups according to the Rotterdam criteria: $A(n=77), B(n=38), C(n=68)$, and $D(n=56)$.

Results: No significant differences were observed between the four PCOS phenotypes for anxiety, depression and QoL, likewise, HRQoL domains related to infertiliy, weight and emotional problems $(P>0.05)$. Phenotypes $A$ and $B$ had worse HRQoL related to hirsutism $(13.98 \pm 5.22,14.13 \pm 6.23, P<0.001)$, also no significant differences were observed between them for HRQoL's domains. While the score of acne in phenotype $D(19.60 \pm 5.12, P=0.003)$ and menstrual score in phenotype $C$ were significantly higher compare with the other PCOS groups $(16.82 \pm 3.87, \mathrm{P}<0.001)$.

Conclusion: Presenting similar psychological profile in all phenotypes unveils the importance of pychological well-being screening, even in milder reproductive phenotypes.

\section{Introduction}

Polycystic ovary syndrome (PCOS) is a frequent endocrine disorder that affects $7.1 \%$ of Iranian reproductive-aged women (1). PCOS is a heterogeneous condition that possesses a remarkably broad spectrum of clinical symptoms and affects many organs systems (2). Additionally, PCOS is accompanied by multiple painful and unfavorable symptoms such as infertility, irregular menstruation, acne, obesity, scalp hair thinning, excess hair growth, which culturally have been defined as unpleasant and unfeminine features and have adverse effects on psychological well-being (3). Hence, these are manifested when finding a partner and sexual activity are important, its cosmetic and psychosexual implications lead to serious emotional distress in affected women (4). Not only might this array of undesirable symptoms have a profound impact on psychological morbidity, but the ranges of treatments that have been offered to relieve them also are a vast contributor to an overall diminished woman's Quality of Life (QoL) (5).

PCOS was diagnosed by Rotterdam criteria which demand two of three characteristics: ultrasound polycystic ovarian morphology (PCOM), hyperandrogenism (HA), and chronic ovulatory dysfunction (OD) (6). Four different phenotypes of PCOS have been introduced by using a possible combination of these criteria: phenotype A includes HA + OD + PCOM, phenotype B and C comprise HA + OD and HA + PCOM respectively, and phenotype D contains OD + PCOM (7). Although, it seems that these different phenotypes of PCOS have regarding hormonal, anthropometric, and metabolic indices (8), there are limited researches to assess reproductive feature and metabolic profile of this diagnostic categories (9). Therefore, it is not completely obvious that these phenotypes suffer from the same negative 
psychological health risk or not, also there is currently no research comparing psychological aspects of different PCOS phenotypes in Iranian papulation.

Given the crucial role of psychological profile in chronic diseases like PCOS because of its impact on selfmanagement, the ability to enhance lifestyle, adherence to therapy, and pursue treatment (10), assessing the psychological status seems to be necessary. The aim of this study was to compare the psychological features such as anxiety, depression, and QoL in women with different PCOS phenotypes.

\section{Material And Methods}

This was a cross-sectional study of women with PCOS who reffered to gynocology clinic of Arash Hospital, Tehran, from 2018 to February 2019 and was approved by the Ethics Committee of Tarbiat Modares University of Medical Sciences (IR.MODARES.REC.1397.153). At first, a sample size was calculated based on taking $95 \%$ confidence interval and $90 \%$ power to test. Finally, 77 patients with phenotype $A, 38$ patients with phenotype $B, 68$ from phenotype $C$, and 56 from phenotype $D$ were entered into the study. All of them were enrolled in the present study via convenience sampling method after obtaining an informed consent.

Overall, Inclusion criteria were: women of reproductive aged (18-45 year), married and non-pregnant, have PCOS based on Rotterdom criteria and willing to participate in the study. Also to avoid possible confounding factors, exclusion criteria included breastfeeding, suffering from chronic diseases (such as diabetes, hypertension...), endocrinological abnormalities (such as primary hyperprolactinemia, thyroid dysfunction, Cushings' syndrome, congenital adrenal hyperplasia, and androgen producing neoplasm), drug and alcohol addiction, taking any antidepressant drugs, and those on hormonal medications for 3 months prior to the study including oral contraceptives, glucocorticoids, ovulation induction agents, and estrogenic or anti-androgenic medications, which might influence on serum androgen levels.

The participants were asked to complete several self-report questionnaire that had been tested for validity and reliability, such as hospital anxiety and depression scale (HADS), Short Form Health Survey (SF-12), and the Modified Polycystic Ovary Syndrome Health-Related Quality of Life Questionnaire (MPCOSQ).

\section{Phenotypical features}

Anthropometric measurements such as body weight, height, hip circumference (HC), and waist circumference (WC) was performed for all participants by a single technician. Body weight $(\mathrm{kg})$ and height $(\mathrm{cm})$ were measured by erect posture and without shoes. Obesity was assessed by Body Mass Index (BMI) which was calculated by dividing the weight $(\mathrm{kg})$ by height $(\mathrm{m})$ square $(11)$. The WC $(\mathrm{cm})$ was masured between the costal margin and the pelvic brim, and $\mathrm{HC}$ at the level of the symphysis pubis. Dividing WC by the $\mathrm{HC}$ was calculated as The waist to hip ratio (WHR)

\section{Clinical features}


All clinical findings such as hirsutism, acanthosis nigricans, galactorrhea, and menstural cycle characteristics were evaluated by single gynechologist. Oligo and anovulation was defined as delaying of menses $>35$ days or $<8$ spontaneous hemorrhagic episodes in a year. clinical hyperandrogenism was measured by as Modified Ferriman Gallwey ( $m-F G)$, which used for scoring the presence of terminal hairs over nine body areas (i.e. upper lip, chin, chest, upper and lower abdomen, thighs, upper and lower back, and upper arms) from 0 to 4 , and $\mathrm{m}-\mathrm{FG}$ score $\geq 8$ is considered hirsutism (12).

\section{Para-clinical assessment}

In the early follicular phase (days 3-5) of spontaneous bleeding or withdrawal bleeding which had been induced by medroxyprogesterone acetate, laboratory assessments and ultrasound sonographies were performed.

All participants underwent abdominal ultrasonography by single sonologist; ovaries containing 12 or more follicles measuring 2-9 mm in diameter and/or enlarged ovarian volume $\left(>10 \mathrm{~mm}^{3}\right)$ on were considered as a positive polycystic sonographic morphology (6).

All blood samples' test was done in Arash hospital laboratory. After overnight bed rest and at least 8 hours fasting. The laboratory investigation consisted of Sex Hormone-Binding Globulin (SHBG), Total Testosterone (TT) levels, and Free Androgen Index (FAl) which was computed by TT (nmol/L)/SHBG $(\mathrm{nmol} / \mathrm{L}) \times 100)$. Hyper androgenism was defined as TT greater than $0.68 \mathrm{ng} / \mathrm{ml}$ or free androgen index greater than $5.36 \%$. All were assessed by commercial kits (Pars Azmoon Inc, Tehran, Iran) using Autoanalyzer BT2000 device. Biochemical measurement of TT and SHBG levels was carried out using Cobas E411 device based on electro-chemiluminescence method (Roche Instr Kit, Germany).

\section{Questionare:}

The participants were asked to complete several self-report questionnaires, as follows:

demographic survey that included the age of the participants, the number of children, their educational levels, occupational status, type of previous deliveries infertility status, and medical history.

\section{Health-Related Quality of Life:}

The Modified Polycystic Ovary Syndrome Health-Related Quality of Life Questionnaire (MPCOSQ) is a valid measure of health-related quality of life (HRQoL) in PCOS patients. It includes 30 seven-point Likert scale questions from six HRQoL areas or domains: emotional disturbances (8items), hirsutism (5 items), infertility (4 items), weight (5 items), menstrual (4items) and acne (4 items). The total score of each field is based on the score obtained in that field divided by the number of questions asked in that field. In sum, largest HRQoL impairment in each field has indicated by a score of 1 , and 7 sugesstes no problem or difficulties(13). The validity and reliability of this questionnaire have been confirmed in Iran(14).

\section{Quality of Life:}


QoL was measured by Short Form Health Survey (SF-12) which containes a total of eight ereas, namely physical functioning, physical role, bodily pain, general health, vitality, social functioning, role emotional, and mental health by 12 questions. Higher scores indicate better QoL (15). The validity and reliability of Iranian version of this questionnaire have been confirmed (16).

\section{Depression and Anxiety:}

The Hospital Anxiety and Depression Scale (HADS) objectively assesses depression and anxiety. This instrument consists of 14 questions (seven item in each subscales) with score range from 0 to 3 . In sum, Higher scores represent higher symptom levels for both subscales and a score of 11 and above is considered a clinical disease (17). The validity and reliability of the Iranian version of HADS have been confirmed (18).

\section{Statistical analysis}

Quantitative variables were presented as Mean \pm Standard Deviation (SD), however, qualitative variables were reported as proportions. First of all, the quantitative variables were assessed for normality using the Kolmogorov-Smirnoff's (KS) test. One-way ANOVA and Kruskal-Wallis (KW) test were applied for the normal and the non-normal/ordinal variables respectively. The Mann-Whitney's $U$ test (MW) performed for pairwise comparison of the group if there was a significant group effect. Then Bonferroni's correction performance $(P<0.008)$ was considered significant. The MW's test was used for compareing normal variables between two PCOS subgroup and qualitative variables were compared by the Chi-square test. Statistical significance was set at $P<0.05$. Data were analyzed by the SPSS software (Statistical Package for the Social Sciences, version 22.0, SPSS Inc., Chicago, IL, USA.)

\section{Result}

Table 1 gives some information about the Basic features of PCOS diagnosis in each group at the beginning of the study. Table 2 showes an overview of demographic characteristics of women with different phenotype of PCOS. The age of the participants ranged from 20 to 43 years. As can be seen, no significant difference was observed between PCOS subgroups in terms of age, BMI, educational level, occupation, WHR, and infertility status $(P>0.05)$. 
Table 1

Basic features of PCOS diagnosis in each group.

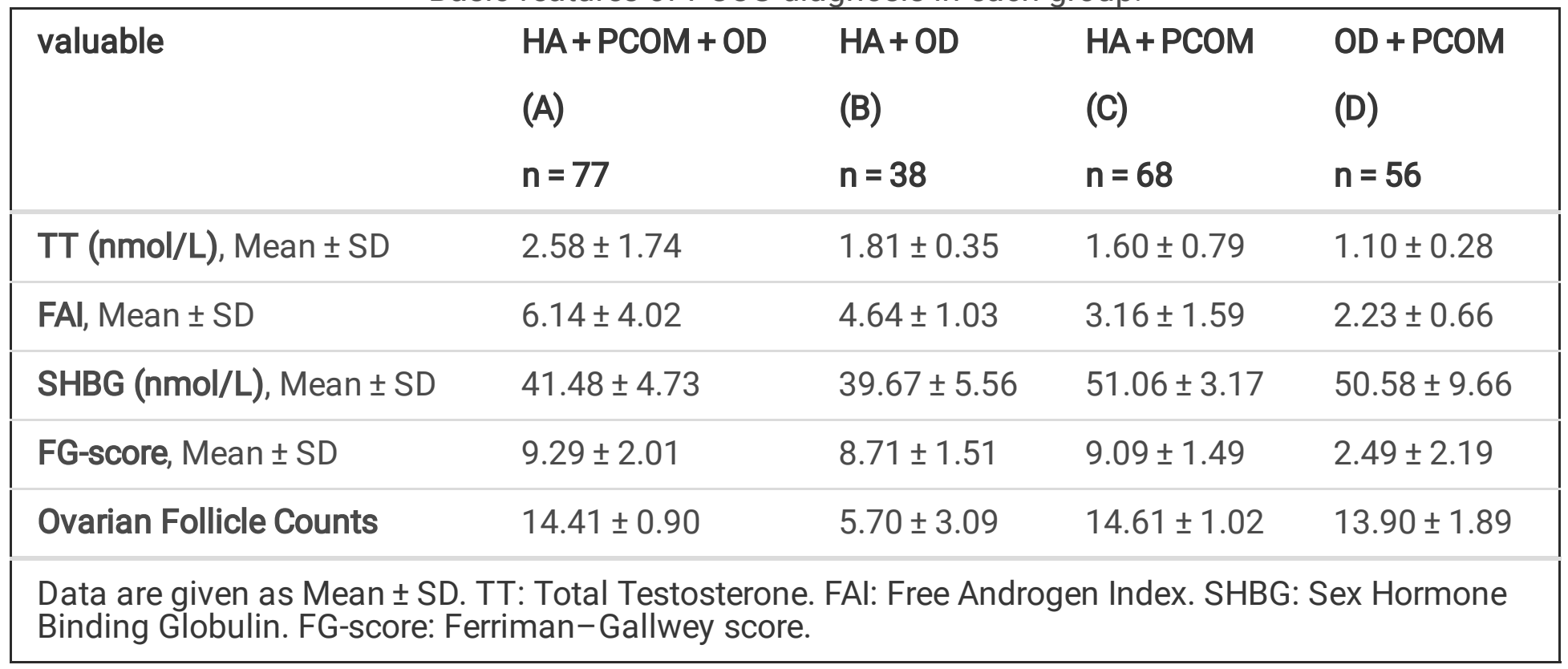


Table 2

Comparison of demographic characteristics between different PCOS phenotypes.

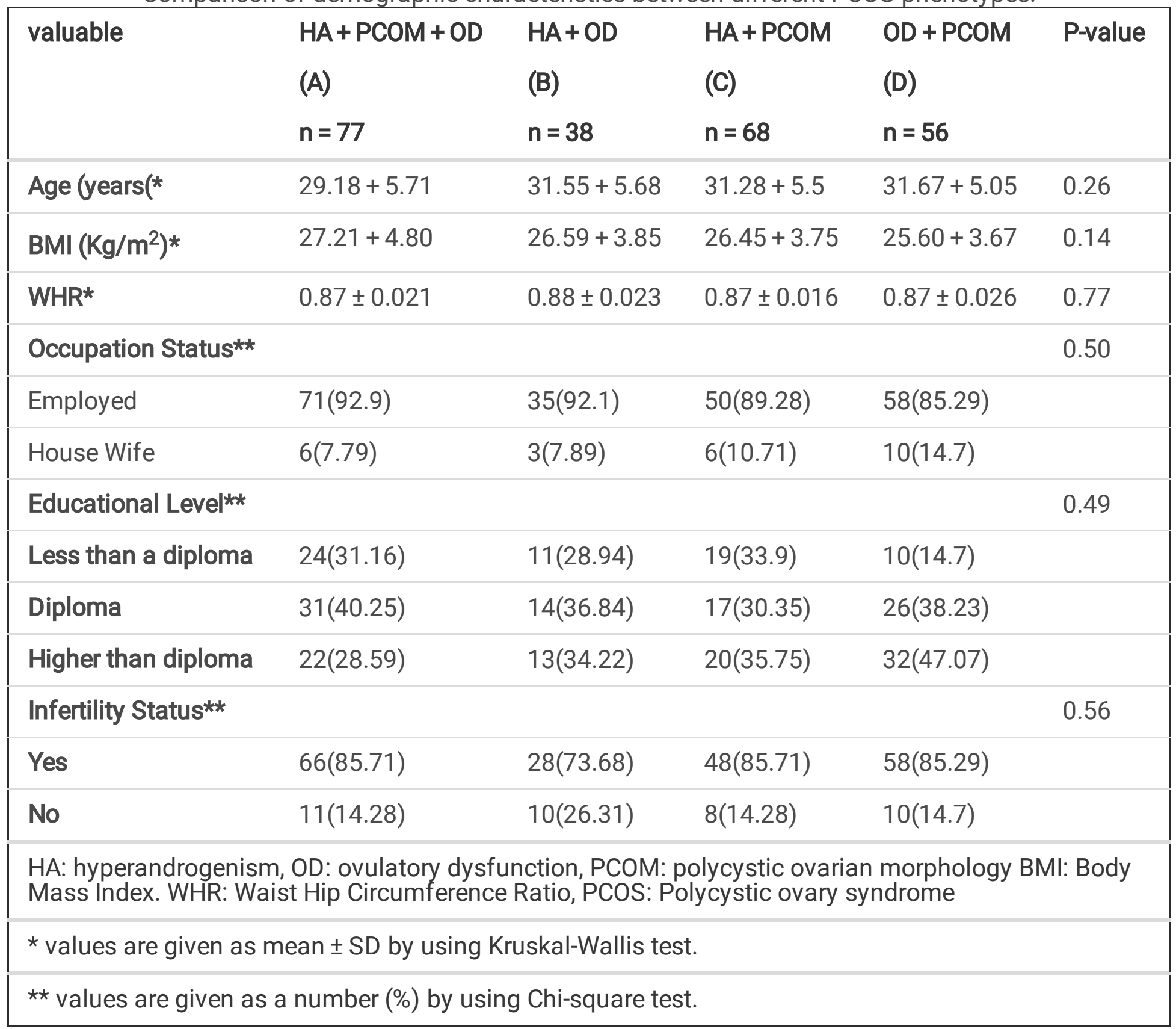

\section{Health-Related Quality of Life:}

Table 3 presents the summery statistics for MPCOSQ in different phenotypes of PCOS. It can be seen that there are no statistically significant differences between different phenotypes categories in the scores of infertility, weight, and emotional disturbance $(P>0.05)$. Additionally, there are no significant differences between phenotype $A$ and $B$ in all domains. Although phenotype $A$ and $B$ indicate lower scores in hirsutism subgroup $(13.98 \pm 5.22,14.13 \pm 6.23$ respectively), phenotype $D$ with $23.33 \pm 4.81$ expresses higher score. Also, in acne and menstrual subgroups phenotype $D$ and $C$ reveal higher scores respectively $(\mathrm{P}<0.001)$. 
Table 3

Comparison of the MPCOSQ scores between different PCOS phenotypes.

\begin{tabular}{|c|c|c|c|c|c|c|}
\hline valuable & $\begin{array}{l}\text { HA + PCOM } \\
+ \text { OD } \\
\text { (A) } \\
n=77\end{array}$ & $\begin{array}{l}H A+O D \\
(B) \\
n=38\end{array}$ & $\begin{array}{l}\mathrm{HA}+\mathrm{PCOM} \\
\text { (C) } \\
\mathrm{n}=68\end{array}$ & $\begin{array}{l}\text { OD + PCOM } \\
\text { (D) } \\
n=56\end{array}$ & $\begin{array}{l}\mathrm{P} \text { - } \\
\text { value* }\end{array}$ & $\begin{array}{l}\text { pair wise } \\
\text { comparison; } \\
\text { P-value** }\end{array}$ \\
\hline \multirow[t]{6}{*}{ Hirsutism } & \multirow[t]{6}{*}{$13.98 \pm 5.22$} & \multirow[t]{6}{*}{$\begin{array}{l}14.13 \pm \\
6.23\end{array}$} & \multirow[t]{6}{*}{$\begin{array}{l}17.22 \pm \\
5.28\end{array}$} & \multirow[t]{6}{*}{$\begin{array}{l}23.33 \pm \\
4.81\end{array}$} & \multirow[t]{6}{*}{$<.001$} & A \&B: 0.75 \\
\hline & & & & & & $A \& D:<0.001$ \\
\hline & & & & & & $A \& C:<0.001$ \\
\hline & & & & & & $B \& D:<0.001$ \\
\hline & & & & & & B\&C: 0.002 \\
\hline & & & & & & D\&C: 0.001 \\
\hline \multirow[t]{6}{*}{ Acne } & \multirow[t]{6}{*}{$17.28 \pm 5.39$} & \multirow{6}{*}{$\begin{array}{l}16.15 \pm \\
5.49\end{array}$} & \multirow{6}{*}{$\begin{array}{l}16.73 \pm \\
4.99\end{array}$} & \multirow{6}{*}{$\begin{array}{l}19.60 \pm \\
5.12\end{array}$} & \multirow[t]{6}{*}{0.003} & A \&B: 0.34 \\
\hline & & & & & & A\&D: 0.007 \\
\hline & & & & & & A\&C: 0.498 \\
\hline & & & & & & B\&D: 0.004 \\
\hline & & & & & & B\&C: 0.668 \\
\hline & & & & & & D\&C: $<0.001$ \\
\hline \multirow[t]{6}{*}{ Menstrual } & \multirow[t]{6}{*}{$12.29 \pm 3.91$} & \multirow{6}{*}{$\begin{array}{l}12.28 \pm \\
3.96\end{array}$} & \multirow{6}{*}{$\begin{array}{l}16.82 \pm \\
3.870\end{array}$} & \multirow{6}{*}{$\begin{array}{l}13.89 \pm \\
4.36\end{array}$} & \multirow{6}{*}{$\begin{array}{l}< \\
0.001\end{array}$} & A\&B: 0.613 \\
\hline & & & & & & A\&D: 0.234 \\
\hline & & & & & & $A \& C:<0.001$ \\
\hline & & & & & & B\&D: 0.48 \\
\hline & & & & & & $B \& C:<0.001$ \\
\hline & & & & & & D\&C: $<0.001$ \\
\hline Weight & $17.90 \pm 6.80$ & $\begin{array}{l}19.23 \pm \\
7.29\end{array}$ & $\begin{array}{l}20.45 \pm \\
7.24\end{array}$ & $\begin{array}{l}19.42 \pm \\
8.08\end{array}$ & 0.15 & - \\
\hline
\end{tabular}

HA: hyperandrogenism, OD: ovulatory dysfunction, PCOM: polycystic ovarian morphology PCOS: Polycystic ovary syndrome

MPCOSQ; Modified Polycystic Ovary Syndrome Health-Related Quality of Life

* P-values refer to the Kruskal-Wallis test

** Kruskal-Wallis test followed by appropriate post hoc test (Mann-Whitney U) (Bonferroni correction was used for multiple comparisons, $\mathrm{P}<0.008$ was considered significant). 


\begin{tabular}{|c|c|c|c|c|c|c|}
\hline valuable & $\begin{array}{l}\text { HA + PCOM } \\
+O D \\
\text { (A) } \\
n=77\end{array}$ & $\begin{array}{l}H A+O D \\
(B) \\
n=38\end{array}$ & $\begin{array}{l}\mathrm{HA}+\mathrm{PCOM} \\
\text { (C) } \\
\mathrm{n}=68\end{array}$ & $\begin{array}{l}\text { OD + PCOM } \\
\text { (D) } \\
n=56\end{array}$ & $\begin{array}{l}\text { P- } \\
\text { value* }\end{array}$ & $\begin{array}{l}\text { pair wise } \\
\text { comparison; } \\
\text { P-value** }\end{array}$ \\
\hline Infertility & $9.85 \pm 4.71$ & $\begin{array}{l}11.26 \pm \\
7.44\end{array}$ & $\begin{array}{l}10.75 \pm \\
5.95\end{array}$ & $9.75 \pm 5.77$ & 0.723 & - \\
\hline Emotional & $19.93 \pm 7.16$ & $\begin{array}{l}20.36 \pm \\
10.02\end{array}$ & $\begin{array}{l}20.95 \pm \\
7.76\end{array}$ & $\begin{array}{l}19.94 \pm \\
8.79\end{array}$ & 0.762 & \\
\hline \multirow[t]{6}{*}{ Total } & \multirow{6}{*}{$\begin{array}{l}91.27 \pm \\
20.87\end{array}$} & \multirow{6}{*}{$\begin{array}{l}93.5 \pm \\
26.53\end{array}$} & \multirow{6}{*}{$\begin{array}{l}102.94 \pm \\
23.11\end{array}$} & \multirow{6}{*}{$\begin{array}{l}105.32 \pm \\
26.28\end{array}$} & \multirow[t]{6}{*}{0.002} & A\&B: 0.91 \\
\hline & & & & & & A\&D: 0.003 \\
\hline & & & & & & A\&C: 0.002 \\
\hline & & & & & & B\&D: 0.021 \\
\hline & & & & & & B\&C: 0.029 \\
\hline & & & & & & D\&C: 0.77 \\
\hline \multicolumn{7}{|c|}{$\begin{array}{l}\text { HA: hyperandrogenism, OD: ovulatory dysfunction, PCOM: polycystic ovarian morphology PCOS: } \\
\text { Polycystic ovary syndrome }\end{array}$} \\
\hline \multicolumn{7}{|c|}{ MPCOSQ; Modified Polycystic Ovary Syndrome Health-Related Quality of Life } \\
\hline \multicolumn{7}{|c|}{ * P-values refer to the Kruskal-Wallis test } \\
\hline
\end{tabular}

\section{Depression and anxiety:}

Table 4 compares the mean scores of different domains of HADS in different phenotypes of PCOS. There are not statistically significant differences between the mean scores of depression, anxiety, and total HADS between different phenotype categories $(P>0.05)$. 
Table 4

Comparing the scores of HADS between different PCOS phenotypes.

\begin{tabular}{|llllll|}
\hline valuable & HA+PCOM + OD & HA+ OD & HA+PCOM & OD + PCOM & P-value* \\
& $(\mathbf{A})$ & $\mathbf{( B )}$ & $\mathbf{( C )}$ & $\mathbf{( D )}$ & \\
& $\mathbf{n}=\mathbf{7 7}$ & $\mathbf{n = 3 8}$ & $\mathbf{n = 6 8}$ & $\mathbf{n = 5 6}$ & \\
\hline Anxiety & $10.74 \pm 4.21$ & $11.73 \pm 3.39$ & $10.50 \pm 3.61$ & $9.82 \pm 4.19$ & 0.245 \\
\hline Depression & $6.74 \pm 3.73$ & $8.43 \pm 3.91$ & $6.37 \pm 3.78$ & $6.29 \pm 3.50$ & 0.092 \\
\hline Total & $17.49 \pm 7.32$ & $20.16 \pm 6.82$ & $16.87 \pm 6.80$ & $16.11 \pm 6.8$ & 0.101 \\
\hline $\begin{array}{l}\text { HA: hyperandrogenism, OD: ovulatory dysfunction, PCOM: polycystic ovarian morphology PCOS: } \\
\text { Polycystic ovary syndrome, HADS: Hospital Anxiety and Depression Scale }\end{array}$ & \\
\hline \multicolumn{4}{|l}{ * P-values are given as mean \pm SD by using Kruskal-Wallis test } \\
\hline
\end{tabular}

\section{Quality of Life:}

Table 5 provieds the results which obtained from summerising the mean scores of QoL domains in PCOS phenotype categories. As shown, there are no ststistically significant differences between phenotype categories $(P>0.05)$. 
Table 5

Comparison of the SF-12 mean scores between different PCOS phenotypes.

\begin{tabular}{|c|c|c|c|c|c|}
\hline Valuable & $\begin{array}{l}\mathrm{HA}+\mathrm{PCOM}+ \\
\mathrm{OD} \\
(\mathrm{A}) \\
\mathrm{n}=77\end{array}$ & $\begin{array}{l}H A+O D \\
(B) \\
n=38\end{array}$ & $\begin{array}{l}\mathrm{HA}+\mathrm{PCOM} \\
\text { (C) } \\
\mathrm{n}=68\end{array}$ & $\begin{array}{l}\text { OD + PCOM } \\
\text { (D) } \\
n=56\end{array}$ & P-value \\
\hline PFX* & $60.90 \pm 31.46$ & $64.16 \pm 26.81$ & $66.51 \pm 30.25$ & $71.56 \pm 28.29$ & 0.338 \\
\hline $\mathrm{RPX}^{*}$ & $55.68 \pm 21.89$ & $54.16 \pm 22.58$ & $54.24 \pm 23.02$ & $64.21 \pm 23.85$ & 0.101 \\
\hline$B P X^{*}$ & $66.81 \pm 24.55$ & $70.83 \pm 28.68$ & $64.28 \pm 25.16$ & $69.60 \pm 25.15$ & 0.491 \\
\hline $\mathrm{GHX}^{*}$ & $50.45 \pm 26.12$ & $36.66 \pm 14.28$ & $48.66 \pm 21.00$ & $45.58 \pm 19.81$ & 0.076 \\
\hline VTX* & $54.09 \pm 24.42$ & $55.00 \pm 24.91$ & $55.80 \pm 24.76$ & $52.94 \pm 27.67$ & 0.914 \\
\hline SFX* & $61.81 \pm 28.40$ & $51.66 \pm 24.50$ & $58.03 \pm 22.91$ & $57.35 \pm 25.63$ & 0.242 \\
\hline REX* & $50.45 \pm 22.68$ & $45.00 \pm 23.80$ & $55.58 \pm 23.22$ & $57.10 \pm 26.36$ & 0.163 \\
\hline $\mathrm{MHX}^{*}$ & $60.68 \pm 22.62$ & $59.58 \pm 16.30$ & $57.36 \pm 18.73$ & $59.80 \pm 19.25$ & 0.865 \\
\hline MCS** & $41.70 \pm 10.40$ & $39.11 \pm 8.83$ & $41.40 \pm 9.66$ & $40.89 \pm 10.18$ & 0.692 \\
\hline PCS** & $43.72 \pm 7.75$ & $43.66 \pm 6.50$ & $43.77 \pm 7.52$ & $45.73 \pm 7.40$ & 0.434 \\
\hline $\begin{array}{l}\text { Total.SF- } \\
12^{\star}\end{array}$ & $546.33 \pm 139.22$ & $\begin{array}{l}519.8 \pm \\
6104.62\end{array}$ & $\begin{array}{l}545.67 \pm \\
128.23\end{array}$ & $\begin{array}{l}564.81 \pm 136+ \\
89\end{array}$ & 0.558 \\
\hline
\end{tabular}

HA: hyperandrogenism, OD: ovulatory dysfunction, PCOM: polycystic ovarian morphology, PCOS: Polycystic ovary syndrome, SF-12: The 12-item Short Form Health Survey, PF: Physical Functioning, RP: Role Physical, BP: Bodily Pain, GH: General Health, VT: Vitality, SF: Social Functioning, RE: Role Emotional, MH: Mental Health, PCS: Physical Component Summary; MCS: Mental Component Summary.

* values are given as mean \pm SD by using Kruskal-Wallis test

** values are given as mean \pm SD by using One-Way ANOVA

\section{Discussion}

PCOS is one of the most common endocrine disorders in women with reproductive age. The adverse impact of diverse clinical manifestations and prolonged complication on worsened QoL and psychological impairments have become a new area of research in the last decades (19). Although it is well-established that PCOS women suffer from anxiety, depression, and markedly reduced QoL $(5,20)$, the association between psychological disorder and each phenotypical category has remained unclear. Therefore, this study was conducted to compare emotional well-being, anxiety and depression between different PCOS phenotypes in Iranian population. 
"Classic" PCOS (Phenotypes A and B):

"Classic "PCOS which include phenotype A and B, had been classified based on chronic OD and HA, also finding PCOM with sonography lead to distinction between these two phenotypes (7). In the current study, comparing different dimension of MPCOSQ across confirmed phenotypic categories of PCOS revealed that classic PCOS represented sever hirsutism symptoms. Also there is not any significant difference between phenotype $A$ and $B$.

First of all, it is important to note that hirsutism has been defined as the excessive terminal hair growth in androgen-dependent areas with male distribution of women body (21). Not only may hirsutism be an unequivocal marker of excessive androgen action (22), but it also influenced by the peripheral metabolism of androgens, insulin resistance and compensatory hyperinsulinism (23). A possible explanation for these results might be the similarity of classic PCOS presentation. Indeed, classic PCOS accompany by abdominal obesity, increased levels of LH and LH/FSH ratio, increased androgens, and elevated insulin and insulin resistance (24).

According to soaring total score of MPCOSQ indicating perturbed health-related QoL, were noted patients with classic PCOS presented more reduction in health-related QoL. These results seem to be consistent with other research which found impaired HRQoL functioning in depressed compared with non-depressed women with PCOS(25). Although, hair growth and menstrual disturbance have revealed as a most distressing factors in Turkish investigation(25), Greenwood et al. (26), suggested infertility and weight problems as the most serious concerns. This inconsistency may be due to clinical compositions and perhaps cultural differences.

"Ovulatory PCOS" (Phenotype C):

Patients with "ovulatory PCOS" generally seems to be the mild form of classic PCOS. Indeed, phenotype C PCOS manifest average values (compared with patients with other phenotypes of PCOS) of serum androgens, insulin, testosterone, atherogenic lipids, hirsutism scores, and prevalence of metabolic syndrome (7). Also Guastella et al. (24) reported intermediate levels of BMI, waist circumference, testosterone in ovulatory PCOS patient compared with classic PCOS and control group. Additionally, normal levels of LH have been described in these patients as the main difference with other phenotypes (24). One of the issues that emerges from these findings is that passaging from classic phenotypes to ovulatory PCOS and vice versa can be related to sociocultural and environmental effects (27).

According to the data from 1,297 Greek women with PCOS have demonstrated that menstrual cycle pattern is more irregular in the "classic" PCOS phenotypes as compared with phenotype $D$, however, its appear to normalize with ageing and in depend from obesity (28).

One unanticipated finding was that phenotype $\mathrm{C}$ patients have reported lower menstrual abnormalities in this study. Also previous investigations revealed discrepant results regarding the relationship between obesity and menstrual cycle abnormalities. Some prior studies have noted that there are not any 
differences between irregular menstruation and obesity $(28,29)$, however, others identified higher rate of menstrual irregularity in overweight women (30).

“Non-hyperandrogenic PCOS” (Phenotype D)

Non-hyperandrogenic PCOS patients have the mildest degree of endocrine and metabolic dysfunction as compared with healthy controls and they have lower LH to FSH ratios, lower total and free T levels, and higher sex hormone-binding globulin levels, as compared with classic PCOS (7).

In the current study, comparing acne in different phenotypes of PCOS showed that the mean score of acne in phenotype $\mathrm{C}$ was higher rather than other phenotypes. This result may be explained by wellestablished fact that androgen levels are increased in women with acne, nevertheless, hirsutism and acne have not any association in women with PCOS $(31,32)$. These discrepancies can be explained by variable local androgen bioactivity (33). MJ Chen et al (34) also demonstrated that presence of acne was related with high level of serum dehydroepiandrosterone sulfate (DHEAS) in PCOS women and a significantly decreased risk of abdominal obesity, independent of serum testosterone concentration and insulin resistance. It is important to mention that in all cases of this study, despite the lack of significant differences between the groups in terms of BMI, WHR, and hormones levels, phenotype $\mathrm{C}$ was associated with lower acne symptoms.

The striking observation to emerge from the data comparison was that the health-related quality of life (HRQoL) in classic phenotype is more impaired as lower total scores. It is important to bear in mind the inconsistency in identifying the most important contributing factor to impaired HRQoL. For instance, Moridi et al. (35) argued that the average of HRQoL and all the six subscales of MPCOSQ in the PCOS women were significantly lower than the healthy group. Similarly, Bazarganipour et al. (36) considered the negative impact of PCOS on HRQoL, although it was not obvious which impairment domains had the most adverse effect as the relative degree of impairment was varied among samples. Nevertheless, in their meta-analysis (37) menstrual irregularities and infertility were reported as the most affected domains in worsened HRQoL than the obesity. Likewise, Yoldemir et al. (38) hold the view that menstrual problems were the worst parameter affecting the QoL.

This study supports evidence from previous observation which evaluated clinical features and HRQoL between infertile women with PCOS phenotypes and women with unexplained infertility (39). The association of Ferriman Gallwey's scores, phenotype classic PCOS, and primary infertility with lower HRQoL were identified (39). Also, Moran et al. (40) who compared different PCOS phenotypes based on National Institute of Health $(\mathrm{NIH})$ criteria (HA and OD), reported poorer HRQoL in women with NIH PCOS compared with non-NIH PCOS. It seems possible that this result is due to the significant effects of clinical HA which (such as excess body hair) on the perception of women as feminine. Hence, psychological wellbeing was reported more related to hirsutism in South Asians rather than white Europeans with PCOS (41). 


\section{Quality Of Life, Depression And Anxiety}

Several studies have confirmed the effectiveness of PCOS on psychological features such as soaring anxiety and depression (42), and worsening QoL (43). This study seeks to obtain data that help to address difference psychological manifestations of PCOS phenotypes. To assess the psychological features, Moran et al. (40) compared different PCOS phenotypes based on National Institute of Health $(\mathrm{NIH})$ criteria $(\mathrm{HA}$ and $\mathrm{OD}$ (. They presented similar anxiety and depression levels in woman with $\mathrm{NIH}$ and non-NIH PCOS. Consistent with the literature, this research found no significant differences between depression, anxiety and various dimension of QoL in different PCOS phenotypes. This emphasizes the intricacy of psychological dysfunction in PCOS. Although, it is difficult to explain this result, it might be related to that PCOS challenges their feminine identity encompassing infertility, acne, hirsutism, and obesity. Consequently, it is highly likely that all these symptoms can possess detrimental effects on QoL and mental health, moreover, potentially precipitate depression and anxiety (44).

The present study has been one of the first attempts to thoroughly perceive differences between PCOS phenotypes in the terms of QoL, HRQoL, depression, and anxiety. Furthermore, it provided an important opportunity to advance the understanding of the requirement of specific mental health screening for each phenotype. Indeed, lifestyle modification as the first-line of beneficial management depends on selfefficacy and psychological well-being. Despite the appropriate study population contrary to the previous studies, this study was limited by the absence of complete information about family history and previous mental illnesses. Further research requires to be carried out in order to estimate female sexual dysfunction in each phenotype's categories and establish the similarities of psychological parameters.

\section{Conclusion}

In this study, it was observed that although presenting similar psychological profiles and QoL scores, phenotypes categories reveal decrement in different in HRQoL's domains.

\section{Abbreviations}

\section{MPCOSQ}

Modified Polycystic Ovary Syndrome Health-Related Quality of Life Questionnaire HADS

hospital anxiety and depression scale

SHBG

Sex Hormone-Binding Globulin

PCOM

Polycystic Ovarian Morphology

HRQOL

Health-Related Quality of Life

DHEAS 
dehydroepiandrosterone sulfate

M-FG

Modified Ferriman Gallwey

PCOS

Polycystic Ovary Syndrome

SF-12

Short Form Health Survey

$\mathrm{NIH}$

National Institute of Health

OD

Ovulatory Dysfunction

FAI

Free Androgen Index

WC

Waist Circumference

BMI

Body Mass Index

WHR

Waist to Hip Ratio

HA

Hyperandrogenism

TT

Total Testosterone

$\mathrm{HC}$

Hip Circumference

MW

Mann-Whitney

QoL

Quality of Life

\section{Declarations}

\section{Ethics approval and consent to participate}

The study was approved by the Ethics Committee of Tarbiat Modares University of Medical Sciences (IR.MODARES.REC. 1397.153). All procedures were in accordance with the ethical standards of the Regional research committee and with the Declaration of Helsinki 1964 and its later amendments.

\section{Consent for publication}

Not applicable. 
Availability of data and materials

The data sets used and analyzed during the current study are available from the corresponding author on reasonable request.

\section{Competing interests}

The authors declare no conflict of interest.

\section{Funding}

None.

\section{Authors' contributions}

Sh.JS and F.B and contributed to the conception and design of the study; Sh.JS, N.M, and F.B did the literature search; Sh.JS, N.M, and F.B performed the statistical analysis; Sh.JS, N.M, and F.B wrote the first draft of the manuscript. All authors contributed to manuscript revision, read, and approved the submitted version.

\section{Acknowledgments}

This study was carried out with the kind collaboration of the participants. This study is a part of research work done in Tarbiat Modares University, Tehran, Iran. There were no conflicts of interest.

\section{References}

1. Tehrani FR, Simbar M, Tohidi M, Hosseinpanah F, Azizi F. The prevalence of polycystic ovary syndrome in a community sample of Iranian population: Iranian PCOS prevalence study. Reproductive Biology Endocrinology. 2011;9(1):1-7.

2. Balen AH, Conway GS, Kaltsas G, Techatraisak K, Manning PJ, West C, et al. Andrology: Polycystic ovary syndrome: the spectrum of the disorder in 1741 patients. Human reproduction. 1995;10(8):2107-11.

3. Naqvi SH, Moore A, Bevilacqua K, Lathief S, Williams J, Naqvi N, et al. Predictors of depression in women with polycystic ovary syndrome. Arch Women Ment Health. 2015;18(1):95-101.

4. Eggers $S$, Kirchengast $S$. The polycystic ovary syndrome-a medical condition but also an important psychosocial problem. Coll Antropol. 2001;25(2):673-85.

5. Jones G, Hall J, Balen A, Ledger W. Health-related quality of life measurement in women with polycystic ovary syndrome: a systematic review. Hum Reprod Update. 2008;14(1):15-25.

6. ESHRE TR, Group A-SPCW. Revised 2003 consensus on diagnostic criteria and long-term health risks related to polycystic ovary syndrome. Fertility sterility. 2004;81(1):19-25. 
7. Lizneva D, Suturina L, Walker W, Brakta S, Gavrilova-Jordan L, Azziz R. Criteria, prevalence, and phenotypes of polycystic ovary syndrome. Fertility sterility. 2016;106(1):6-15.

8. Pehlivanov B, Orbetzova M. Characteristics of different phenotypes of polycystic ovary syndrome in a Bulgarian population. Gynecological endocrinology. 2007;23(10):604-9.

9. Moran L, Teede H. Metabolic features of the reproductive phenotypes of polycystic ovary syndrome. Hum Reprod Update. 2009;15(4):477-88.

10. Goldney RD, Phillips PJ, Fisher LJ, Wilson DH. Diabetes, depression, and quality of life: a population study. Diabetes Care. 2004;27(5):1066-70.

11. Who EC. Appropriate body-mass index for Asian populations and its implications for policy and intervention strategies. Lancet. 2004;363(9403):157.

12. Escobar-Morreale H, Carmina E, Dewailly D, Gambineri A, Kelestimur F, Moghetti P, et al. Epidemiology, diagnosis and management of hirsutism: a consensus statement by the Androgen Excess and Polycystic Ovary Syndrome Society. Hum Reprod Update. 2012;18(2):146-70.

13. Cronin L, Guyatt G, Griffith L, Wong E, Azziz R, Futterweit W, et al. Development of a health-related quality-of-life questionnaire (PCOSQ) for women with polycystic ovary syndrome (PCOS). The Journal of Clinical Endocrinology Metabolism. 1998;83(6):1976-87.

14. Bazarganipour F, Ziaei S, Montazeri A, Faghihzadeh S, Frozanfard F. Psychometric properties of the Iranian version of modified polycystic ovary syndrome health-related quality-of-life questionnaire. Human reproduction. 2012;27(9):2729-36.

15. Ware JE Jr, Kosinski M, Keller SD. A 12-Item Short-Form Health Survey: construction of scales and preliminary tests of reliability and validity. Medical care. 1996:220-33.

16. Montazeri A, Vahdaninia M, Mousavi SJ, Omidvari S. The Iranian version of 12-item Short Form Health Survey (SF-12): factor structure, internal consistency and construct validity. BMC Public Health. 2009;9(1):341.

17. Snaith RP. The hospital anxiety and depression scale. Health Qual Life Outcomes. 2003;1(1):1-4.

18. Montazeri A, Vahdaninia M, Ebrahimi M, Jarvandi S. The Hospital Anxiety and Depression Scale (HADS): translation and validation study of the Iranian version. Health Qual Life Outcomes. 2003;1(1):14.

19. Teede H, Deeks A, Moran L. Polycystic ovary syndrome: a complex condition with psychological, reproductive and metabolic manifestations that impacts on health across the lifespan. BMC Med. 2010;8(1):41.

20. Månsson M, Holte J, Landin-Wilhelmsen K, Dahlgren E, Johansson A, Landén M. Women with polycystic ovary syndrome are often depressed or anxious-a case control study.

Psychoneuroendocrinology. 2008;33(8):1132-8.

21. Escobar-Morreale HF. Diagnosis and management of hirsutism. Ann N Y Acad Sci. 2010;1205(1):166-74. 
22. Goodarzi MO, Shah NA, Antoine HJ, Pall M, Guo X, Azziz R. Variants in the 5a-reductase type 1 and type 2 genes are associated with polycystic ovary syndrome and the severity of hirsutism in affected women. The Journal of Clinical Endocrinology Metabolism. 2006;91(10):4085-91.

23. Landay M, Huang A, Azziz R. Degree of hyperinsulinemia, independent of androgen levels, is an important determinant of the severity of hirsutism in PCOS. Fertility sterility. 2009;92(2):643-7.

24. Guastella E, Longo RA, Carmina E. Clinical and endocrine characteristics of the main polycystic ovary syndrome phenotypes. Fertility sterility. 2010;94(6):2197-201.

25. Cinar N, Kizilarslanoglu MC, Harmanci A, Aksoy DY, Bozdag G, Demir B, et al. Depression, anxiety and cardiometabolic risk in polycystic ovary syndrome. Hum Reprod. 2011;26(12):3339-45.

26. Greenwood EA, Pasch LA, Cedars MI, Legro RS, Huddleston HG, Network HDRM, et al. Association among depression, symptom experience, and quality of life in polycystic ovary syndrome. American journal of obstetrics gynecology. 2018;219(3):279. e1-. e7.

27. Di Fede G, Mansueto P, Longo RA, Rini G, Carmina E. Influence of sociocultural factors on the ovulatory status of polycystic ovary syndrome. Fertility sterility. 2009;91(5):1853-6.

28. Panidis D, Tziomalos K, Papadakis E, Chatzis P, Kandaraki EA, Tsourdi EA, et al. Associations of menstrual cycle irregularities with age, obesity and phenotype in patients with polycystic ovary syndrome. Hormones. 2015;14(3):431-7.

29. Cupisti S, Kajaia N, Dittrich R, Duezenli H, Beckmann MW, Mueller A. Body mass index and ovarian function are associated with endocrine and metabolic abnormalities in women with hyperandrogenic syndrome. European journal of endocrinology. 2008;158(5):711-20.

30. Strowitzki T, Capp E, von Eye Corleta $\mathrm{H}$. The degree of cycle irregularity correlates with the grade of endocrine and metabolic disorders in PCOS patients. European Journal of Obstetrics Gynecology Reproductive Biology. 2010;149(2):178-81.

31. Karrer-Voegeli S, Rey F, Reymond MJ, Meuwly J-Y, Gaillard RC, Gomez F. Androgen dependence of hirsutism, acne, and alopecia in women: retrospective analysis of 228 patients investigated for hyperandrogenism. Medicine. 2009;88(1):32-45.

32. Chuan SS, Chang RJ. Polycystic ovary syndrome and acne. Skin Therapy Lett. 2010;15(10):1-4.

33. Cappel M, Mauger D, Thiboutot D. Correlation between serum levels of insulin-like growth factor 1 , dehydroepiandrosterone sulfate, and dihydrotestosterone and acne lesion counts in adult women. Archives of dermatology. 2005;141(3):333-8.

34. Chen M-J, Chen C-D, Yang J-H, Chen C-L, Ho H-N, Yang W-S, et al. High serum dehydroepiandrosterone sulfate is associated with phenotypic acne and a reduced risk of abdominal obesity in women with polycystic ovary syndrome. Human reproduction. 2011;26(1):227-34.

35. Moridi M, Rahebi S. Health related quality of life in women with polycystic ovary syndrome. Iranian Journal of Reproductive Medicine. 2015.

36. Bazarganipour F, Taghavi SA, Montazeri A, Ahmadi F, Chaman R, Khosravi A. The impact of polycystic ovary syndrome on the health-related quality of life: A systematic review and metaanalysis. Iranian journal of reproductive medicine. 2015;13(2):61. 
37. Bazarganipour F, Ziaei S, Montazeri A, Foroozanfard F, Faghihzadeh S. Health-related quality of life and its relationship with clinical symptoms among Iranian patients with polycystic ovarian syndrome. Iranian Journal of Reproductive Medicine. 2013;11(5):371.

38. Yoldemir T, Angin P, Ramoglu S, Atasayan K. Health-related quality of life (HRQL) in women with polycystic ovary syndrome (PCOS). Maturitas. 2017;100:175.

39. Dilbaz B, Çınar M, Özkaya E, Tonyalı NV, Dilbaz S. Health related quality of life among different PCOS phenotypes of infertile women. Journal of the Turkish German Gynecological Association. 2012;13(4):247.

40. Moran LJ, Deeks A, Gibson-Helm M, Teede HJ. Psychological parameters in the reproductive phenotypes of polycystic ovary syndrome. Human reproduction. 2012;27(7):2082-8.

41. Kumarapeli V, Seneviratne RdA, Wijeyaratne C. Health-related quality of life and psychological distress in polycystic ovary syndrome: a hidden facet in South Asian women. BJOG: An International Journal of Obstetrics Gynaecology. 2011;118(3):319-28.

42. Barry JA, Kuczmierczyk AR, Hardiman PJ. Anxiety and depression in polycystic ovary syndrome: a systematic review and meta-analysis. Human reproduction. 2011;26(9):2442-51.

43. Castelo-Branco C, Naumova I. Quality of life and sexual function in women with polycystic ovary syndrome: a comprehensive review. Gynecol Endocrinol. 2020;36(2):96-103.

44. Podfigurna-Stopa A, Luisi S, Regini C, Katulski K, Centini G, Meczekalski B, et al. Mood disorders and quality of life in polycystic ovary syndrome. Gynecological endocrinology. 2015;31(6):431-4. 